\title{
Atividades lúdicas para promoção de saúde bucal em escolares: revisão de literatura
}

\author{
Playful activities to promote oral health in schoolchildren: literature review
}

Actividades lúdicas para promover la salud bucal en escolares: revisión de la literatura

Paulla Valéria de Souza Meneses ${ }^{1 *}$, Érica Paula Barbosa ${ }^{1}$, Flávia Accioly Canuto Wanderley ${ }^{1}$, Carmen Silvia Motta Bandini'.

\section{RESUMO}

Objetivo: Avaliar se as atividades lúdicas em saúde bucal realizadas com escolares contribuem para mudança de hábitos em relação à higiene oral. Métodos: Revisão sistemática da literatura mediante consulta a: Biblioteca Virtual de Saúde (BVS), Literatura Latino-americana e do Caribe em Ciências da Saúde (Lilacs) e Biblioteca Eletrônica Científica Online (Scielo), utilizando os descritores: higiene bucal, saúde bucal, educação em saúde bucal, placa dentária, escolares, jogos, brinquedos, recreação, jogos educativos e jogos infantis. Foram considerados elegíveis estudos experimentais, com crianças em idade escolar (5 a 12 anos). Resultados: Foram selecionados 186 estudos elegíveis, recuperados pela busca de forma pareada e independente por dois pesquisadores, retiradas 57 duplicatas e após a aplicação dos critérios de elegibilidade na leitura dos títulos e resumos houve discordâncias entre os dois pesquisadores, que foram resolvidas por um terceiro avaliador, permanecendo 4 estudos. As intervenções educativas lúdicas promoveram mudanças nos indicadores de higiene oral (índice de placa visível e índice de sangramento gengival), o que sugere melhorias nos comportamentos relacionados à saúde bucal. Considerações finais: A promoção em saúde bucal em escolares impõe desafios e, diferentes intervenções educativas lúdicas podem ser capazes de promover mudanças favoráveis de comportamento relacionados à saúde bucal.

Palavras-chave: Saúde bucal, Educação em saúde bucal, Placa dentária, Escolares, Recreação.

\begin{abstract}
Objective: To evaluate whether recreational activities in oral health carried out with schoolchildren contribute to changing habits in relation to oral hygiene. Methods: Systematic literature review by consulting: Virtual Health Library (VHL), Latin American and Caribbean Literature in Health Sciences (Lilacs) and Online Scientific Electronic Library (Scielo), using the keywords: oral hygiene, oral health, oral health education, dental plaque, school children, games, toys, recreation, educational games and children's games. Experimental studies with school-age children (5 to 12 years) were considered eligible. Results: 186 eligible studies were selected, retrieved by the search in a paired and independent manner by two researchers, 57 duplicates were removed and after the application of the eligibility criteria in reading the titles and abstracts, there were disagreements between the two researchers, which were resolved by a third party. evaluator, remaining 4 studies. Playful educational interventions promoted changes in oral hygiene indicators (visible plaque index and gingival bleeding index), which suggests improvements in behaviors related to oral health. Final considerations: The promotion of oral health in schoolchildren imposes challenges and different playful educational interventions may be able to promote favorable behavioral changes related to oral health.
\end{abstract}

Keywords: Oral health, Oral health education, Dental plaque, Schoolchildren, Recreation.

1 Universidade Estadual de Ciências da Saúde de Alagoas (UNCISAL), Maceió - AL.

*E-mail: paullavaleria@hotmail.com 


\section{RESUMEN}

Objetivo: Evaluar si las actividades recreativas en salud bucal realizadas con escolares contribuyen al cambio de hábitos en relación a la higiene bucal. Métodos: Revisión sistemática de la literatura mediante consultas: Biblioteca Virtual en Salud (BVS), Literatura Latinoamericana y del Caribe en Ciencias de la Salud (Lilacs) y Biblioteca Electrónica Científica en Línea (Scielo), utilizando las palabras clave: higiene bucal, salud bucal, educación en salud bucal, placa dental, escolares, juegos, juguetes, recreación, juegos educativos y juegos infantiles. Los estudios experimentales con niños en edad escolar (5 a 12 años) se consideraron elegibles. Resultados: Se seleccionaron 186 estudios elegibles, recuperados por la búsqueda de manera pareada e independiente por dos investigadores, se eliminaron 57 duplicados y luego de la aplicación de los criterios de elegibilidad en la lectura de títulos y resúmenes, existieron desacuerdos entre los dos investigadores, los cuales fueron resueltos por un tercero. evaluador, 4 estudios restantes. Las intervenciones educativas lúdicas promovieron cambios en los indicadores de higiene bucal (índice de placa visible e índice de sangrado gingival), lo que sugiere mejoras en los comportamientos relacionados con la salud bucal. Consideraciones finales: La promoción de la salud bucal en los escolares impone desafíos y diferentes intervenciones educativas lúdicas pueden ser capaces de promover cambios conductuales favorables relacionados con la salud bucal.

Palabras clave: Salud bucal, Educación en salud bucal, Placa dental, Escolares, Recreación.

\section{INTRODUÇÃO}

O Global Burden of Disease Study em 2010, traz a distribuição e as causas de várias doenças e fatores de risco à saúde e aponta que doenças bucais estão presentes em cerca de 3,9 bilhões de pessoas no mundo, sobressaindo-se a cárie em dentes permanentes que acontece em $35 \%$ da população (MARCENES K, et al., 2013).

Macedo LR, et al. (2017) afirmam que a saúde bucal é parte fundamental e indissociável da saúde geral, no entanto, atualmente, a cárie dental e a doença periodontal ainda são consideradas as enfermidades bucais mais frequentes, constituindo-se um problema de saúde pública que pode resultar na perda do elemento dental, se atenção precoce e adequada não Ihes for direcionada (GARCIA PPNS, et al., 2009).

O biofilme dental é um dos principais fatores etiológicos dessas doenças bucais, constituindo-se no acúmulo de bactérias colonizadoras da cavidade oral na superfície dos dentes. Sua presença está diretamente relacionada aos hábitos de higiene oral do paciente e o seu controle, a redução dos índices de cárie dental e de doença periodontal (MORAES KR, et al., 2011; SILVA AS, et al., 2011).

Essas doenças são altamente dependentes de comportamentos que são socialmente construídos e, por isso, trazem a necessidade de implantação de atividades educativas, visando a sua prevenção e estimulando a adoção de comportamentos de autonomia, bem como favorecer o conhecimento dos indivíduos sobre 0 processo saúde doença (ALVES GG e AERTS D, 2011).

A higiene oral inadequada, que leva a comportamentos prejudiciais e nocivos para a saúde, principalmente em crianças, é causada por falta de instrução e informação corretas. Porém, só informar não é o suficiente para mudar hábitos, é necessária uma relação dialogal, em que os participantes possam estar envolvidos em todos os momentos de ações educativas (TSUZUKI FM, et al., 2007).

Além de ensinar as crianças pequenas diretrizes sobre escovação e o papel da dieta nas intervenções bucais, é essencial desenvolver seu interesse em aprender hábitos para manter uma boa saúde bucal por toda a vida (MAHESWARI UM, et al., 2014).

A promoção em saúde bucal exige permanente mudança de estratégias e métodos, para impactar seus efeitos a longo prazo, alterando positivamente comportamentos e hábitos. Por meio de ações de promoção da saúde, pode-se captar o indivíduo e ensiná-lo a melhorar a própria saúde, tornando-o empoderado, possibilitando a transformação desse sujeito, em ator principal do autocuidado (KUSMA SZ, et al., 2012). 
Assim a educação em saúde torna-se um elemento chave no desenvolvimento de programas de promoção da saúde bucal, visto que é o processo pelo qual as crianças constroem seus conhecimentos sobre a importância da saúde bucal, assim como também é promovido o desenvolvimento das habilidades necessárias para que elas possam atingir e manter uma saúde bucal adequada, além de prevenir as doenças bucais que são mais prevalentes nessa população (MACEDO LR, et al., 2017).

Nesse sentido, a escola tem se mostrado como um ambiente propício para a construção de hábitos de higiene bucal. Projetos de educação em saúde, principalmente em escolares, têm proporcionado o desenvolvimento de melhores técnicas de escovação e uso de fio dental, tão importantes para manter os dentes hígidos (GARBIN CAS, et al., 2013; OLIVEIRA MF, et al., 2019).

Programas de saúde escolar são importantes para promover a saúde bucal de crianças e jovens uma vez que os alunos podem ser acessados facilmente durante os anos escolares, período que vai da infância à adolescência. São anos influentes em que comportamentos relacionados à saúde ao longo da vida, assim como estilo de vida e atitudes, estão sendo desenvolvidos. As crianças são predominantemente favoráveis durante esse período e, quanto mais cedo os hábitos são estabelecidos, o impacto é mais duradouro (SADANA G, et al., 2017).

Garcia PPNS, et al. (2009) relatam que alguns autores acreditam ser a idade escolar uma época adequada para a realização de programas educativo-preventivos, uma vez que crianças e adolescentes estão mais dispostos a adquirir novos conhecimentos, respondem com maior eficiência ao treinamento, apresentam maior interesse devido à influência do próprio grupo e, por estarem em ambiente escolar, onde o treinamento e controle são mais adequados, torna-se possível o desenvolvimento e aprendizado de hábitos de higiene bucal de forma correta.

Contudo, Angelopoulou MV, et al. (2015) destacam que educação em saúde bucal nas escolas, por meio de palestras tradicionais, obtém sucesso apenas na melhoria do conhecimento em saúde bucal, enquanto tem baixa eficácia na higiene bucal e na saúde gengival, sugerindo que outras estratégias são necessárias no sentido de mudança de hábitos e comportamentos.

Reforçando os achados de Angelopoulou MV, et al. (2015), em estudo de revisão, Lumkukl I, et al. (2015) concluíram que as intervenções analisadas para educação em saúde não se preocuparam em incorporar uma visão ampliada do modelo de promoção da saúde e a efetividade delas na prevenção ou na redução de diferentes agravos bucais não pôde ser claramente demonstrada.

Outro aspecto a ser considerado reside na necessidade de que as ações preventivas sejam iniciadas no ambiente familiar e consolidadas no ambiente escolar. Nesse ambiente, os comportamentos adequados são fixados e reforçados pelos professores. Estes, além de responsáveis pela disseminação do conhecimento, exercem papel ativo na solidificação dos hábitos saudáveis que acompanharão o indivíduo por toda a vida. A influência que exercem sobre as crianças deve ser considerada decisiva para o estabelecimento de condutas e hábitos saudáveis (BARRETO DM, et al., 2013).

Assim sendo, verifica-se a necessidade de práticas educacionais direcionadas para cada idade, que sensibilizem as crianças no tocante às doenças causadas pelos maus hábitos de higiene e alimentação e que se voltem também para a prevenção de tais doenças, permitindo que os pais participem, pois, o envolvimento da família se torna primordial para a perpetuação das práticas de educação e saúde. Embora a maioria das crianças perceba a importância da higiene bucal, estas não reconhecem o papel da dieta para manutenção da saúde, o que vai ao encontro da falta de preparo dos educadores para orientá-los, havendo a necessidade de maior integração escola-dentista (MACEDO LR, et al., 2017).

A motivação é muito importante para o sucesso na orientação de higiene oral em pré escolares, não devendo ser aplicada uma única vez, reforços motivacionais, através de outras sessões, em programas educativos preventivos atuam positivamente para a redução do biofilme dental e sangramento gengival (MORAES KR, et al., 2011; TOASSI RFC e PETRY PC, 2002).

O profissional de saúde que deseja ter sucesso em sua intenção de ensinar deve estar estimulado e em sintonia com seu público-alvo para conseguir motivar e impulsionar mudanças. $O$ interesse e a curiosidade despertados facilitam a aquisição de comportamentos positivos de saúde (CAMPESTRINI NTF, et al., 2019). 
Menegaz AM, et al. (2018) destacam que as atividades educacionais devem ser personalizadas, baseadas em teorias comportamentais e, que além dos aspectos individuais, necessitam considerar o contexto sociocultural e as relações interpessoais dos sujeitos. Além desses aspectos, os autores reforçam que a qualidade destas ações educacionais é mais importante do que os recursos pedagógicos que utilizam.

Tratando-se de um público infantil, é importante que as atividades sejam prazerosas, sendo o lúdico um facilitador desse processo. A ludicidade, enquanto método educativo para promoção da saúde bucal infantil, tem se mostrado eficaz, pois as atividades lúdicas, quando sistematicamente planejadas e corretamente desenvolvidas, funcionam atraindo a atenção e gerando motivação da criança no processo de aprendizagem e estimulando a mudança ou adoção de comportamento que possam melhorar a sua qualidade de vida (ANTÔNIO LP, et al., 2015; COTA ALS e COSTA BJA, 2017).

Dentre essas práticas lúdicas, podemos citar o estudo de Malik A, et al. (2017), numa escola primária na Índia, que aplicou a educação em saúde bucal baseada em jogos e verificaram que o estado de saúde bucal das crianças pode ser melhorado se a promoção de saúde nas escolas for realizada de maneira abrangente e interessante.

Um indicativo de sucesso das intervenções para prevenção de doenças bucais pode advir do efeito positivo em relação aos comportamentos em saúde bucal, visto que para sua manutenção faz-se necessária a construção de comportamentos saudáveis (MENEGAZ AM, et al., 2018).

Sendo assim, esse estudo se propõe a realizar uma revisão bibliográfica a fim de avaliar se as atividades lúdicas em saúde bucal realizadas com escolares contribuem para mudança de hábitos em relação à higiene oral.

\section{MÉTODOS}

Foi realizada uma busca sistemática da literatura nas bases de dados BVS, Lilacs e Scielo.

A pergunta da pesquisa foi estruturada no formato do acrônimo PICO, sendo $\mathrm{P}$, o paciente ou sujeitos da pesquisa, I, a intervenção realizada no estudo, C, a comparação efetuada e O, os "outcomes", ou seja, os resultados ou desfecho. No caso do presente estudo, P são os escolares, I a introdução de recurso educativo lúdico nas atividades de saúde bucal, $\mathbf{C}$ atividade educativas convencionais e $\mathbf{O}$ a mudança de hábito.

Os termos de busca combinaram descritores em ciências da saúde (DeCS) e operadores booleanos, usando a seguinte estratégia: (higiene bucal) OR (saúde bucal) AND (educação em saúde bucal) AND (placa dentária) AND (escolares) AND (jogos) OR (brinquedos) OR (recreação) OR (jogos educativos) OR (jogos infantis). A busca foi realizada entre outubro de 2019 e março de 2020.

Foram definidos os critérios de elegibilidade que incluíram na revisão estudos: a) experimentais, cuja variável independente tenha sido o uso de atividades lúdicas, como jogos, brincadeiras, entre outras e que a variável dependente estivesse relacionada à mudança no comportamento, quanto aos hábitos de higiene oral; b) crianças em idade escolar na c) faixa etária de 5 a 12 anos. Foram excluídos os estudos apenas descritivos, teóricos ou de revisão bibliográfica, em que não houve uma intervenção. Não foram limitados ano ou língua de publicação.

Todos os dados foram tabulados em planilhas do programa Excel e serão apresentados de forma descritiva, relacionando dados quantitativos e qualitativos.

\section{RESULTADOS}

Foram identificados 186 estudos elegíveis recuperados pela busca, selecionados de forma pareada e independente por dois pesquisadores. Desses, 134 foram encontrados na BVS, 48 na Lilacs e 4 na Scielo. Foram retiradas da amostra 57 duplicatas, restando 129 estudos. Após a aplicação dos critérios de elegibilidade mediante leitura dos títulos e resumos, o primeiro pesquisador elegeu três estudos, e o segundo pesquisador cinco estudos. Tal situação requereu um terceiro avaliador para ajudar na decisão das discordâncias, após a avaliação deste terceiro juiz a amostra final foi composta por 5 artigos elegíveis para revisão. Desses, apenas quatro estavam disponíveis de forma integral, sendo excluído 1 estudo, por indisponibilidade da versão completa do artigo original (Figura 1). 
Figura 1 - Fluxograma de seleção dos estudos.

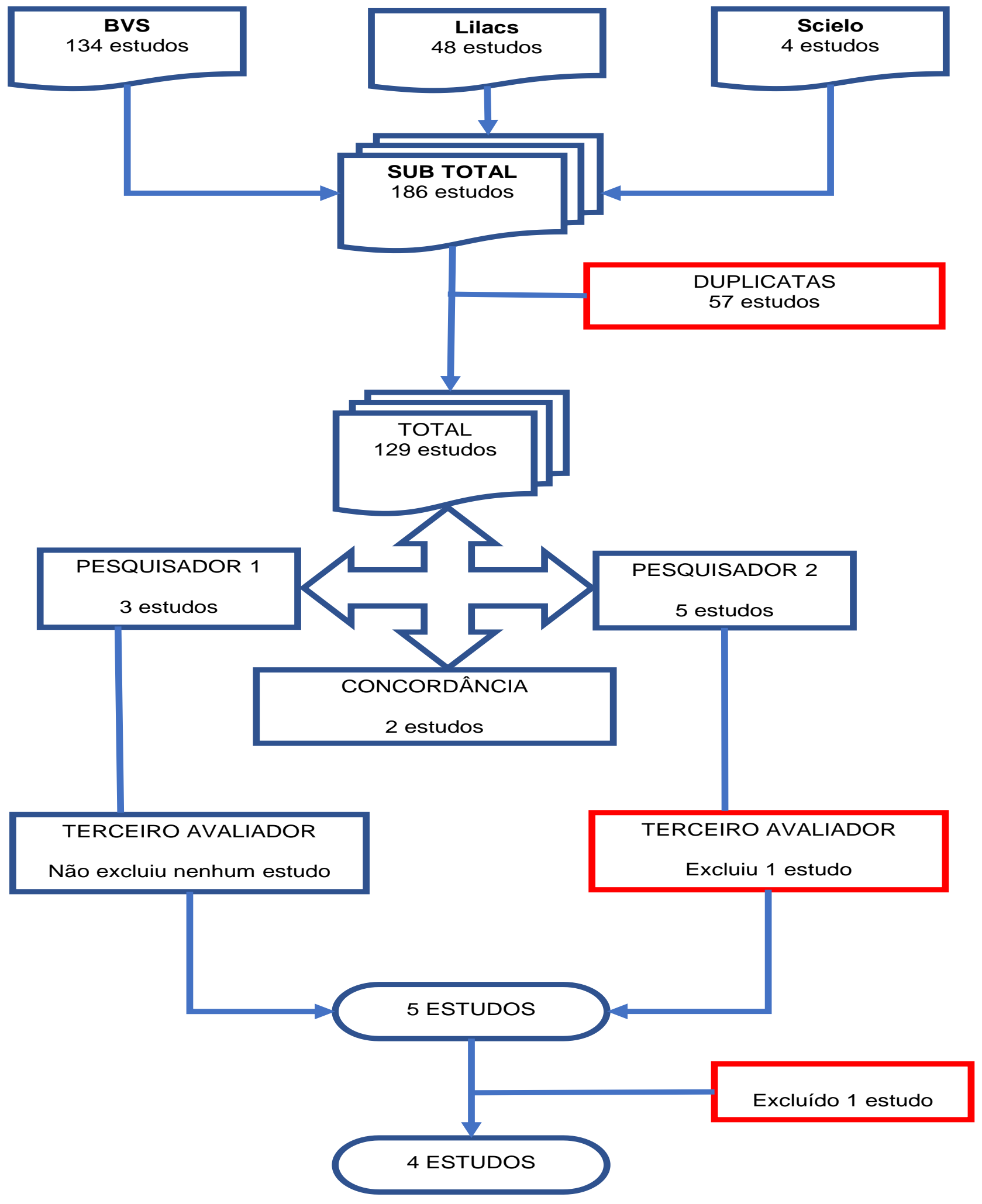

Fonte: Meneses PVS, et al., 2020.

Os quatro estudos foram classificados por autor, ano, tipo de estudo, participantes, exposição, intervenção, desfecho e tempo de acompanhamento, para extração dos dados, conforme a tabela a seguir, que resume as principais características dos estudos incluídos na revisão (Quadro 1). 
Quadro 1 - Extração de dados dos estudos.

\begin{tabular}{|c|c|c|c|c|}
\hline $\begin{array}{c}\text { AUTOR } \\
\text { ANO DE } \\
\text { PUBLICAÇÃO }\end{array}$ & $\begin{array}{l}\text { BARRETO DM, } \\
\text { et al., } 2013\end{array}$ & $\begin{array}{l}\text { GAMBOA F, et } \\
\text { al., } 2018\end{array}$ & $\begin{array}{l}\text { GARCIA PPNS, } \\
\text { et al., } 2009\end{array}$ & $\begin{array}{l}\text { MAHESWARI UM, } \\
\text { et al., } 2014\end{array}$ \\
\hline TIPO DE ESTUDO & $\begin{array}{l}\text { Estudo piloto de } \\
\text { ensaio clínico } \\
\text { randomizado }\end{array}$ & $\begin{array}{l}\text { Prospectivo } \\
\text { longitudinal }\end{array}$ & Experimental & Experimental \\
\hline PARTICIPANTES & $\begin{array}{l}38 \text { crianças } \\
3-5 \text { anos }\end{array}$ & $\begin{array}{l}23 \text { crianças } \\
3-7 \text { anos }\end{array}$ & $\begin{array}{l}120 \text { crianças } \\
8-10 \text { anos }\end{array}$ & $\begin{array}{l}120 \text { crianças } \\
5-10 \text { anos }\end{array}$ \\
\hline EXPOSIÇÃO & $\begin{array}{c}\text { Grupo Controle: } \\
\text { não houve } \\
\text { intervenção. } \\
\text { Grupo } \\
\text { Experimental: } \\
\text { teatro de } \\
\text { fantoches, } \\
\text { brincadeiras } \\
\text { interativas e } \\
\text { cartazes. }\end{array}$ & $\begin{array}{l}\text { Entrevistas, } \\
\text { aulas, palestras, } \\
\text { oficinas } \\
\text { educativas e } \\
\text { atividades } \\
\text { recreativas. }\end{array}$ & $\begin{array}{c}\text { Grupo 1: } \\
\text { controle } \\
\text { Grupo 2: } \\
\text { leitura } \\
\text { Grupo 3: } \\
\text { discussão } \\
\text { Grupo 4: } \\
\text { reescrita do } \\
\text { material de auto } \\
\text { instrução. }\end{array}$ & $\begin{array}{c}\text { Grupo A: } \\
\text { cartão de memória } \\
\text { Grupo B: } \\
\text { jogos combinados } \\
\text { com cartões de } \\
\text { memória. }\end{array}$ \\
\hline INTERVENÇÃO & $\begin{array}{l}\text { Sessão única de } \\
60 \text { minutos de } \\
\text { duração, com } \\
\text { coleta de dados: } \\
\text { antes e } 30 \text { dias } \\
\text { após intervenção. }\end{array}$ & $\begin{array}{c}\text { Atividades } \\
\text { educativas a } \\
\text { cada } 15 \text { dias, } \\
\text { durante } 6 \\
\text { meses, com } \\
\text { coleta de dados } \\
1 \text { dia antes } \\
\text { (linha de base), } \\
1 \text { e } 6 \text { meses } \\
\text { após processo } \\
\text { educativo. }\end{array}$ & $\begin{array}{l}\text { Sessão única, } \\
\text { com coleta de } \\
\text { dados antes e } \\
\text { após } 30 \text { dias da } \\
\text { intervenção. }\end{array}$ & $\begin{array}{c}\text { Sessões } 1 \text { vez ao } \\
\text { dia, durante } 7 \text { dias, } \\
\text { com coleta de } \\
\text { dados antes, } 1 \text { e } 3 \\
\text { meses após } \\
\text { intervenção. }\end{array}$ \\
\hline DESFECHO & $\begin{array}{c}\text { Redução do IPV e } \\
\text { ISG. }\end{array}$ & $\begin{array}{c}\text { Redução } \\
\text { significativa nos } \\
\text { níveis de S. } \\
\text { mutans na } \\
\text { saliva de } \\
\text { crianças com } \\
\text { cárie. }\end{array}$ & Redução no IPV. & $\begin{array}{c}\text { Aumento } \\
\text { significativo dos } \\
\text { bons escores de } \\
\text { higiene oral. }\end{array}$ \\
\hline $\begin{array}{c}\text { TEMPO DE } \\
\text { ACOMPANHAMENTO }\end{array}$ & 30 dias & 6 meses & 30 dias & 3 meses \\
\hline
\end{tabular}

Legenda: IPV - Índice de Placa Visível; ISG - Índice de sangramento Gengival; S. mutans - Streptococcus mutans. Fonte: Meneses PVS, et al., 2020.

Uma outra análise, procurou identificar a área de conhecimento da revista, a área de atuação dos autores e o país de publicação dos estudos selecionados (Tabela 2).

Tabela 2 - Extração de dados das revistas.

\begin{tabular}{|l|c|c|c|}
\hline \multicolumn{1}{|c|}{ AUTOR } & $\begin{array}{c}\text { ÁREA DE } \\
\text { CONHECIMENTO }\end{array}$ & ÁREA DOS AUTORES & PAís \\
\hline BARRETO DM, et al., 2013 & Ensino, Saúde & Odontologia & Brasil \\
\hline GARCIA PPNS, et al., 2009 & Saúde & Odontologia & Brasil \\
\hline GAMBOA F, et al., 2018 & Saúde & Odontologia, ciências, imunologia & Colômbia \\
\hline MAHESWARI UM, et al., 2014 & Saúde & Odontologia, & Índia \\
\hline
\end{tabular}

Fonte: Meneses PVS, et al., 2020. 


\section{DISCUSSÃO}

Dada a dimensão e importância do assunto, poucos foram os estudos encontrados. Percebe-se que há uma carência de pesquisas na área de educação sobre o emprego de ludicidade para promoção de saúde bucal com escolares. Existem poucos educadores pesquisando essas ações, as revistas onde os estudos foram publicados, não são voltados para educação, prevalecendo as da área da saúde, tanto no Brasil como fora dele.

Em um estudo piloto Barreto DM, et al. (2013), dividiram 38 crianças em dois grupos: experimental (E), que participou de atividades educativas e preventivas por 60 minutos (teatro com fantoches, brincadeiras interativas, macromodelos, cartazes e escovação dental supervisionada); e controle (C), que não recebeu a referida atividade durante o período do estudo. Os autores verificaram índice de placa visível (IPV), índice de sangramento gengival (ISG) e experiência de cárie, antes e 4 semanas após a intervenção. No entanto, os dados sobre a frequência dessa intervenção e como as atividades foram realizadas não são claramente descritos.

Barreto DM, et al. (2013) trouxeram a participação dos pais como reforço do trabalho junto as crianças, favorecendo, assim, todo o núcleo familiar com as ações executadas, corroborando o que é descrito na literatura por Garbin CAS, et al. (2015), que reconhecem no reforço no lar, principalmente pelos pais, um dos grandes sucessos para os programas de promoção da saúde bucal.

Outro estudo incluído nessa revisão, que promoveu reforço no lar, com envolvimento dos pais foi o realizado por Gamboa F, et al. (2018), em Bogotá, Colômbia. De maneira prospectiva e longitudinal, avaliaram a presença e contagem de Streptococcus mutans, em amostras de saliva de crianças com cárie, na linha de base do estudo, aos 3 e 6 meses após um processo de educação em saúde bucal envolvendo os pais e os escolares. A contagem de Streptococcus mutans teve redução significativa em $64,8 \%$ e $86,6 \%$ em 3 e 6 meses, respectivamente, em comparação com os níveis encontrados antes do processo educacional.

Os dados coletados por Barreto BM, et al. (2013), através de questionário estruturado, respondido pelos pais, mostraram que a escovação era um hábito diário, porém o fio dental não era utilizado por $84,2 \%$ e 52,6\% das crianças dos grupos $\mathrm{C}$ e $\mathrm{E}$ (atividades educativas e preventivas), respectivamente. O Instituto Brasileiro de Geografia e Estatística (IBGE) em 2015, constatou que um pouco mais da metade da população brasileira $(53 \%)$ usava os três itens fundamentais para uma boa higiene oral: escova de dentes, creme dental e fio dental. O IPV e ISG inicial foram similares entre os grupos, já ao final do estudo, observou-se uma diferença significativa entre eles, com redução dos índices no grupo E (IPV 40,31 - 15,24; ISG 3,16 - 0) e aumento no C (IPV 35,04 - 43,63: ISG 4,93 - 7,67), evidenciando que atividades educativas preventivas realizadas com o grupo E ajudaram na qualidade da higiene oral realizada (BARRTETO BM, et al., 2013).

Além disso, por se tratar de um estudo piloto, o estudo de Barreto BM, et al. (2013) apresenta algumas limitações, tais como: a amostra por conveniência não é representativa de uma dada população e, dessa forma, não podem os resultados serem extrapolados para a população escolar e o curto período da intervenção (30 dias) não permite avaliar como os hábitos adquiridos se comportam ao longo do tempo, sugere-se a realização de estudos de avaliação prospectiva por um período maior.

A motivação das crianças durante o processo educativo envolve a utilização de um número diversificado de atividades e recursos, em que as crianças reagem de forma diferente aos diversos estímulos trabalhados (ANTÔNIO LP, et al., 2015).

Tal fato foi observado por Garcia PPNS, et al. (2009), ao utilizar um mesmo método sob diferentes enfoques. Participaram do estudo crianças de quatro séries de um mesmo ano escolar, divididas em quatro grupos e submetidas a intervenções diferentes para observar os níveis de higiene oral, mediante análise do índice de placa antes e após a aplicação dos métodos educativos. As atividades educativas dividiram-se em leitura, discussão e complete (reescrita da história) de um mesmo material (gibi): grupo 1 - controle; grupo 2 - leitura; grupo 3 - discussão e grupo 4 - complete (reescrita da história).

Após avaliação em 2 períodos, antes dos métodos educativos e decorridos 30 dias, houve uma melhoria geral no índice de placa dos quatro grupos avaliados, com uma porcentagem maior de queda nos grupos 
onde houve discussão $(41,4 \%)$ e complete $(34,8 \%)$, sugerindo que a leitura do gibi foi meramente informativa e que os maiores percentuais de queda aconteceram nos grupos onde o gibi teve seu conteúdo trabalhado, no entanto, não houve diferença estatisticamente significativa entre os quatro grupos, transcorridos 30 dias (GARCIA PPNS, et al., 2009).

O índice de placa de O'Leary usado no estudo experimental, de Garcia PPNS, et al. (2009) difere daquele usando por Barreto DM, et al. (2013) o IPV, porém ambos avaliam a qualidade da higiene oral e os estudos assemelham-se no tempo entre as coletas de dados inicial e final (30 dias) e o grupo controle não recebeu nenhum tipo de intervenção.

Um outro estudo experimental, conduzido por Maheswari UM, et al. (2014), na Índia, buscou comparar a eficácia da educação em saúde bucal convencional e a baseada em jogos com conhecimento relacionado à saúde bucal e o status de higiene bucal. Para isso 120 crianças de 5 a 10 anos, foram divididas em 2 grupos: grupo A, recebeu educação em saúde bucal através de cartões de memória e grupo $B$ através de brincadeiras, combinadas com cartões de memória. Cada grupo teve 30 crianças de 5 a 7 anos e 30 crianças de 8 a 10 anos, que foram expostas a intervenção 1 vez por dia, durante 7 dias.

As avaliações em relação a higiene oral, do estudo acima, usaram o Índice de Higiene Oral Simplificada (IHOS), registrados antes da intervenção, 1 dia e 3 meses após a intervenção. Finalizado o estudo verificouse que no grupo B (5-7 e 8-10) e no grupo A (8-10) houve um aumento significativo de bons escores de higiene bucal com queda dos escores regulares e ruins, nas três avaliações durante o estudo. Evidenciando que a depender do tipo de intervenção utilizada e da idade da população estudada pode-se obter resultados diferentes nos comportamentos e, consequentemente, na mudança de hábitos (MAHESWARI UM, et al., 2014).

Isso reforça os achados por Angelopoupou MV, et al. (2015), que teve como principal resultado que o efeito do aprendizado experiencial contribuiu para redução da placa dentária, gengivite e cárie em comparação com o método tradicional de palestras e como resultados secundários teve-se a melhoria do conhecimento, comportamento e atitude em saúde bucal. Ele coloca que o aprendizado por meio de experiência é uma abordagem educacional em que o aprendizado ocorre através da experiência e este auxilia no aprimoramento do conhecimento e na atitude na educação em saúde e recentemente foi introduzido na educação em saúde bucal com resultados promissores

Na sua maioria, os artigos encontrados, Barreto BM, et al. (2013), Maheswari UM, et al. (2014) e Garcia PPNS, et al. (2009), procuraram avaliar o índice de placa por ser ela um dos principais fatores etiológicos da cárie e do sangramento gengival, são índices que podem indicar a qualidade da higiene oral realizada. No entanto, para avaliar a experiência de cárie e mudança de hábitos de higiene oral que levaram a sua redução, conforme realizado por Gamboa F, et al. (2018) e Garcia PPNS, et al. (2009), é necessário que o estudo tenha um período maior, pois o seu desenvolvimento ou redução não consegue ser observado em intervalo curto de tempo (BARRTETO BM, et al., 2013).

Estudos como o de Gamboa F, et al. (2018), não apresentam fácil reprodutibilidade e dependem de estrutura laboratorial e familiaridade com as técnicas de análise empregadas. Os autores coletaram 3 amostras de saliva espontânea, por sucção suave, com pipetas estéreis. Após a coleta as amostras foram diluídas com tampão de fosfato $0,05 \mathrm{M}$, por fim inoculadas em placas de Petri, com incubação a $37^{\circ} \mathrm{C}$ por 48 horas, após o crescimento bacteriano foram contadas as colônias de bactérias e expressas em unidades formadoras de colônias (UFC) por $\mathrm{ml}$ de saliva e realizada a identificação das cepas bacterianas. Esses recursos nem sempre estão disponíveis para aqueles que não estão no meio acadêmico. Outro fator a ser considerado são as práticas educativas aplicadas que se basearam em palestras, workshops e oficinais, não havendo intervenção direta relacionada a prática de higiene oral.

Não foi encontrada muita similaridade nos métodos utilizados pelos estudos selecionados. Observou-se variação no tipo de intervenção, tempo de acompanhamento, técnicas empregadas, estratégias de ensino e quantidade de grupos. Não foi possível, dessa forma, avaliar qual das intervenções poderia ter melhores resultados e qual o tempo ideal para aplicação. Nesse sentido, há a necessidade de estudos futuros com maior qualidade metodológica e embasamento teórico das intervenções, bem como descrição mais detalhada do método e intervenções. 
Menegaz AM, et al. (2018) destacam que para se verificar o sucesso em desfechos clínicos de saúde bucal, como a cárie, os estudos devem considerar o tamanho da amostra, a dosagem da intervenção (número de contatos com a população-alvo) e o tempo de acompanhamento. Esses fatores metodológicos tornam-se importantes para os efeitos positivos na redução de doença, gerados pela mudança de comportamentos.

Nesta revisão a heterogeneidade metodológica das intervenções não possibilitou a realização de síntese estatística através da metanálise, constituindo-se num limitador, assim como a busca não ter sido feita em todas as bases de dados.

\section{CONSIDERAÇÕES FINAIS}

Diferentes intervenções educativas podem ser capazes de promover mudanças favoráveis de comportamento relacionados à saúde bucal. Essas mudanças positivas foram observadas através de indicadores de higiene oral, como o IPV e ISG, que servem para avaliar a motivação do indivíduo em realizar a higiene bucal, o autocuidado e a melhoria na higiene. No entanto, a heterogeneidade dos estudos, não permitiu avaliar qual o melhor tipo de intervenção e se a mudança de hábitos perdurou, sendo assim, faz-se necessário mais estudos experimentais e com maior abrangência, a fim de estabelecer qual intervenção apresenta melhores resultados, como também, avaliações por um período maior para verificar como os hábitos adquiridos se comportam ao longo do tempo.

\section{REFERÊNCIAS}

1. ALVES GG, AERTS D. As práticas educativas em saúde e a Estratégia Saúde da Família. Cien Saúde Colet, 2011; 16(1): 319-25.

2. ANGELOPOULOU MV, et al. Comparative clinical study testing the effectiveness of school based oral health education using experiential learning or traditional lecturing in 10 year old children. BMC Oral Health, 2015; 15(51): 1-7.

3. ANTÔNIO LP, et al. Avaliação de diferentes métodos educativos em saúde bucal em crianças na faixa etária de 7 a 10 anos de idade. RFO, 2015; 20(1): 52-58.

4. BARRETO DM, et al. Avaliação da eficácia de uma atividade educativo-preventiva com pré-escolares: estudo piloto de um ensaio clínico randomizado. Arq Odontol, 2013; 49(3): 113-121.

5. CAMPESTRINI NTF, et al. Atividades educativas em saúde bucal desenvolvidas por cirurgião dentista com escolares: uma revisão sistematizada da literatura. Revista da ABENO, 2019; 19(4): 46-54.

6. COTA ALS, COSTA BJA. Atividades lúdicas como estratégia para promoção da saúde bucal infantil. Revista Saúde e Pesquisa, 2017; 10(2): 365-371.

7. GAMBOA F, et al. Presence and count of $S$. mutans in children with dental caries: before, during and after a processo f oral health education. Acta Odontol. Latinoam., 2018;31(3): 156-163.

8. GARBIN CAS, et al. Conhecimento em saúde bucal e práticas desenvolvidas por professores do ensino fundamental. RFO, 2013; 18(2): 321-27.

9. GARBIN CAS, et al. Oral health education in school: parent's attitudes and prevalence of caries in children. Rev. Odontol. UNESP, 2015; 44(5): 285-291.

10. GARCIA PPNS, et al. Educação em Saúde: Efeito de um Método de Auto Instrução sobre os Níveis de Higiene Oral em Escolares. Pesq Bras Odontoped Clin Integr, 2009; 9(3): 333-337.

11. IBGE. Instituto Brasileiro de Geografia e Estatística. Pesquisa Nacional de Saúde: 2013. Acesso e utilização dos serviços de saúde, acidentes e violências: Brasil, grandes regiões e unidades da federação. Rio de Janeiro: Coordenação de Trabalho e Rendimento, 2015.

12. KUSMA SZ, et al. Promoção de saúde: perspectivas avaliativas para saúde bucal na atenção primária em saúde. Cad. Saúde Pública, 2012; 18: 9-19.

13. LEMKUHL I, et al. A efetividade das intervenções educativas em saúde bucal: revisão de literatura. Cad. Saúde Colet., 2015; 23(3): 336-346.

14. MACEDO LR, et al. Promoção de saúde bucal para pré escolares: relato de experiência. Rev. Ciênc. Ext., 2017; 13(4): 128-139.

15. MAHESWARI UN, et al. Effects of Conventional vs Game-based Oral Health Education on Children's Oral Healthrelated Knowledge and Oral Hygiene Status - A Prospective Study. Oral Health \& Preventive Dentistry, 2014; 12(4): 331-336.

16. MALIK A, et al. Implementation of Game-based Oral Health Education vs Conventional Oral Health Education on Children's Oral Health-related Knowledge and Oral Hygiene Status. Int J of Clin Pediatr Dent, 2017; 10(3): 257-260.

17. MARCENES K, et al. Global burden of oral conditions in 1990-2010: a systematic analysis. J Dent Res, 2013; $92(7)$ : 592-597.

18. MENEGAZ AM, et al. Intervenções educativas em serviços de saúde e saúde bucal: revisão sistemática. Rev Saúde Pública, 2018; 52 (52): 1-14. 
19. MORAES KR, et al. Motivação de higiene dental utilizando brinquedos com temas odontológicos. ConScientiae Saúde, 2011; 19(4): 723-728.

20. OLIVEIRA MF, et al. Avaliação do índice de placa visível antes e depois de sessões de educação em saúde bucal com crianças. Rev. Aten. Saúde, 2019; 17(60): 37-46.

21. SADANA G, et al. Evaluation of the Impact of Oral Health Education on Oral Hygiene Knowledge and Plaque Control of School-going Children in the City of Amritsar. J Int Soc Prev Community Dent, 2017; 7(5): 259-263.

22. SILVA AS, et al. Controle mecânico do biofilme dental. Revista Gestão \& Saúde, 2011; 2(2): 1-6.

23. TOASSI RFC, PETRY PC. Motivação no controle do biofilme dental e sangramento gengival em escolares. Rev Saúde Pública, 2002; 36(5): 634-637.

24. TSUZUKI FM, et al. Prevalência e severidade da cárie dentária em um município em condições de vulnerabilidade social no estado do Paraná. Arch health invest, 2007; 11(22): 281-294. 